\title{
A METHOD FOR DIGITAL QUANTIFICATION OF EOSINOPHILS INFILTRATE IN ORAL SQUAMOUS CELL CARCINOMA
}

\author{
Rania H. Younis ${ }^{1,2,3}$, B.D.S., M.D.S., Ph.D.
}

\begin{abstract}
INTRODUCTION:The recent advances in tumor immunotherapy triggered more attention towards analysis of tumor inflammation. Oral squamous cell carcinoma (OSCC) is malignancy that can be therapeutically challenging and resistant to treatment. Further investigation of the inflammatory profile in OSCC can enhance our understanding of the disease and hence patient response to immunotherapy. Eosinophils are suggested to play a significant role in tumor progression and have been described to be associated with advanced stage malignancies of several tumor subtypes.

OBJECTIVE: Here we wanted to assess a methodology to quantitatively measure eosinophils infiltrate in OSCC.

MATERIAL AND METHODS: Eosinophils infiltrate was analyzed in formalin fixed paraffin embedded tissue (FFPE) microarray cores of 30 oral squamous cell carcinomas (OSCC), and 10 normal oral epithelium controls. Digital analysis using the auto fluorescence of eosinophils accentuated by Alexa Fluor 488 stain, in FFPE tissue sections; we used Cytation 5 machine and Gen 5 software, to digitally quantify the extent of eosinophils infiltrate in OSCC.

RESULTS: Out of the inflamed OSCC examined sections with heavy inflammatory infiltrate rich in eosinophils, RBCs, neutrophils and monocytes, eosinophils were readily detected, and digitally quantified after staining with Alexa Fluor 488. The tumor cores showed eosinophils infiltrate in the peri-tumoral stroma of 26 (87\%) of the 30 examined OSCC

CONCLUSION: This study highlights the importance of assessment of the type and extent of inflammatory cells infiltrate as well as its digital quantification. It provides a novel quantification method of eosinophils that opens avenues to study the correlation between eosinophils infiltrate and tumor prognosis.

KEY WORDS: digital, quantification, eosinophils, oral squamous cell carcinoma, autofluorescence

RUNNING TITLE: Digital quantification of eosinophils in oral squamous cell carcinoma.
\end{abstract}

1 Lecturer, Department of Oral Pathology, Faculty of Dentistry, Alexandria University, Egypt.

2 Assistant professor of oral pathology, Department of Oncology and Diagnostic Sciences, School of Dentistry, University of Maryland Baltimore.

3 Associate member, University of Maryland Greenebaum Comprehensive Cancer Center, Tumor immunology and immunotherapy division.

*Corresponding author:

rania.younis@gmail.com

\section{INTRODUCTION}

Head and neck squamous cell carcinoma (HNSCC) is an aggressive malignancy that affects the lining mucosa of the pharynx, larynx, oral cavity, and sinus lining. It can be substantially invasive and destructive of the head and neck area. The overall five years survival rate is $\sim 50-60 \%$. The main etiological factor is smoking. Alcohol has a synergistic effect, while human papilloma virus (HPV) has been described as an etiological factor specifically in oropharyngeal type of HNSCC $(1,2)$. Oral squamous cell carcinoma (OSCC) is capable of escaping immune surveillance through several mechanisms that induce recruitment of immune suppressive inflammatory cells $(3,4)$. The recent advent in HNSCC immunotherapy is promising, yet it is limited by several factors including but not limited to immunotherapy toxic effects, allergic reactions, as well as resistance to immunotherapy acquired through immune suppressor cells $(1,5)$. Better understanding of the tumor inflammatory profile can improve our understanding of patients' response to immunotherapy.

Immune suppression has been described in HNSCC since the 1995 (6). The tumor cells induce a complex machinery of immune suppressor cells that work together to support tumor progression. The immune suppressor cells in tumor associated inflammation bears similarities to other pathologic conditions, specifically the immune profile under allergic and parasitic infections. Eosinophils are a main player in such conditions (7). Although the role of eosinophils in allergic reactions and parasitic infections is well described (8), the extent of eosinophils infiltrate and their role in malignancy is poorly understood.

Eosinophils develop from granulocyte monocyte precursor cells, the same cells that give rise to mast cells. They are bi-nucleated cells with abundant granular eosinophilc cytoplasm and are 6-10um in dimension (9). It has been described in the tumor tissue in several malignancies including head and neck squamous cell carcinoma (10-13). The quantification of eosinophils infiltrate is a promising mechanism by which we can estimate their potential role in tumor immunity and the extent of tumor mediated immune suppression $(9,14)$. Here we present a novel methodology of digital quantification of eosinophils in formalin fixed paraffin embedded tissue (FFPE) sections of OSCC and two of the most common 
salivary gland tumors using eosinophils auto fluorescence, accentuated by Alexa Fluor 488, and read under Cytation 5 cell imaging multi mode reader machine.

\section{MATERIAL AND METHODS}

\section{Tumor tissue}

The formalin fixed paraffin embedded (FFPE) tissue microarray (TMA) of Oral cavity tumor tissue, T 273, HOraC080PG01, and HN802 purchased from US Biomax, was analysed. Thirty cases of OSCC, and 10 normal oral epithelial tissue control were included in the study.

\section{Tissue staining}

TMA was processed similar to routine immunofluorescence technique. Briefly, tissue sections were deparaffinized in xylene, rehydrated in graded ethanol, treated with Tris- EDTA buffer for antigen retrieval, and quenched in hydrogen peroxide to block endogenous peroxidase. Tissue sections were blocked with $2.5 \%$ normal plasma, overnight at $4{ }^{0} \mathrm{C}$, then stained with antibody antimouse IgG Alexa Fluor 488 (catalog no. A-11001) (Thermo Fischer, Rockford, IL) for 30 minutes in room temperature, and mounted in DAPI (4',6-Diamidino-2-Phenylindole (double stranded DNA staining) mounting media.

Analysis

H\&E was viewed using aperio image scope soft ware. The eosinophils auto fluorescence accentuated using the Alexa Fluor 488 was viewed using the Bioteck Cytation 5 cell imaging multi-mode reader.

\section{RESULTS}

\section{Eosinophils infiltrate in a sample of oral malignancy}

The oral tumor tissue cores of TMA were examined for eosinophils infiltrate. The tumor cores showed eosinophils infiltrate in the peri-tumoral stroma of 26 (87\%) of the 30 examined OSCC. The examined SCC stroma was heavily inflamed with mononuclear cells, eosinophils and neutrophilic infiltrate (Fig 1 A-C). Examined normal epithelium was generally negative for eosinophils except for few noticed in some peripheral blood vessels.

Eosinophils Fluorescence observed in OSCC.

Eosinophils were previously described in the peritumoral stroma of oral SCC using subjective description and counting $(10,15)$. Flow cytometry allows for digital counting using the inherent auto fluorescence of eosinophils in peripheral blood (16). To investigate if eosinophils can be digitally quantified using its inherent fluorescence in FFPE tumor tissue sections, while preserving the spatial tissue configuration, we stained a serial section of the same TMA examined on H\&E, using Alexa Fluor 488 to accentuate any endogenous auto-fluorescence, then viewed the tissue using cytation 5 cell imaging multimode reader. The examined tissue cores showed scattered fluorescent cells that can be delineated using the Cytation 5 software excluding the background tissue fluorescence (Fig. 2 A-B). Higher power confirmed that the fluorescent cells were scattered cells in the peri-tumoral stroma of 6-10um in size (Fig. 2 C-D). To investigate the type of fluorescent cells, DAPI nuclear stain was used. The DAPI stain illustrated that the florescent cells were bi-nucleated cells consistent with eosinophils (Fig 3 AC). Examining the corresponding H\&E serial section confirmed eosinophils in the area.

\section{Digital quantification of eosinophils fluorescence in the} peri-tumoral stroma of SCC

The cytation 5 software allows for digital quantification of the fluorescence set at a specific intensity. We used the software to align the eosinophils, then set the intensity of the cells as the level to quantify. This allows the software to subtract any background auto-fluorescence of lower intensity (Fig. 4A). This provided a quantitative measure of the eosinophils infiltrate in the SCC stroma (Fig. 4B), to compare with the normal oral mucosa that was almost negative compared to the OSCC.

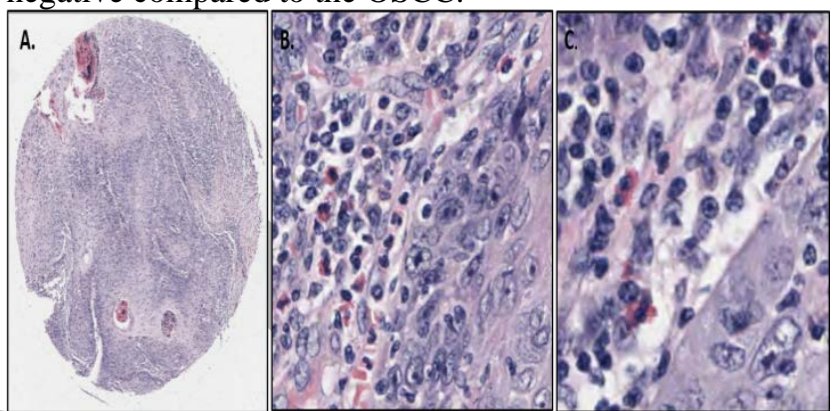

Figure 1: Inflamed SCC peri-tumoral stroma. A. H\&E stained well differentiated SCC tissue core $(2 x)$. B. Inflamed tumor stroma shows mix of monocytic cells, and eosinophils infiltrate (40x). C. 20x magnification of the same section as in B confirms eosinophil infiltrate.

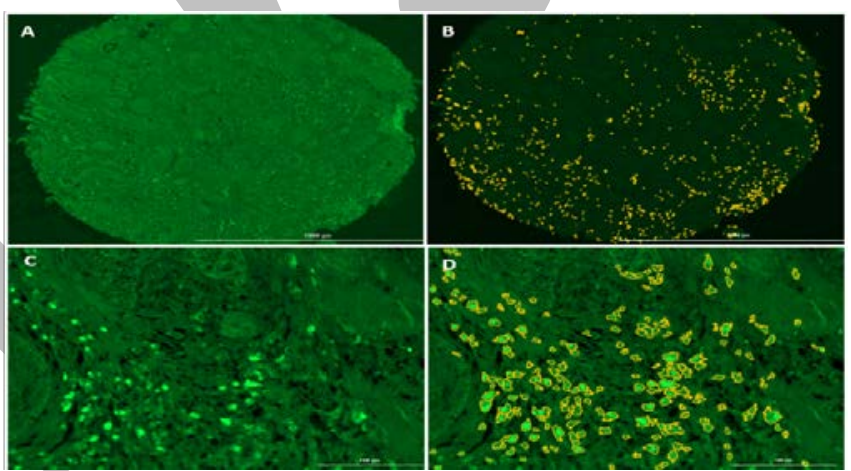

Figure 2: Squamous cell carcinoma of the lower lip stained using Alexa Fluor 488. A. Scattered Alexa Fluor 488 auto fluorescence accentuated inflammatory cells. B. The fluorescent inflammatory cells can be delineated using the quantitative analysis tool of cytation 5 cell imaging multimode reader after excluding background tissue fluorescence (2x). C. Higher power Digital alignment confirms the auto fluorescence is scattered cells in the peri-tumoral stroma. D. Demarcation of the fluorescent eosinophils using the cytation 5 software. C and D, Cells examined at 20x, 100um scale bar.

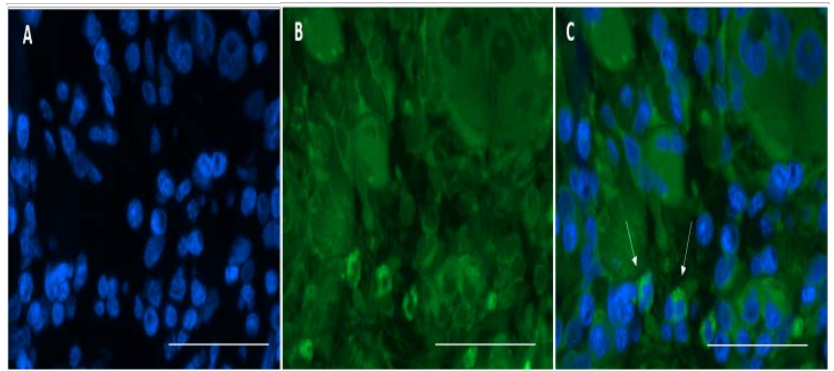

Figure 3: The fluorescent cells show bi-lobed nucleus, as examined on higher magnification. A. DAPI nuclear stain. B. 
Alexa Fluor 488 stain. C. Merge of Alexa Fluor 488 and DAPI. (40x, 100um scale bar ).
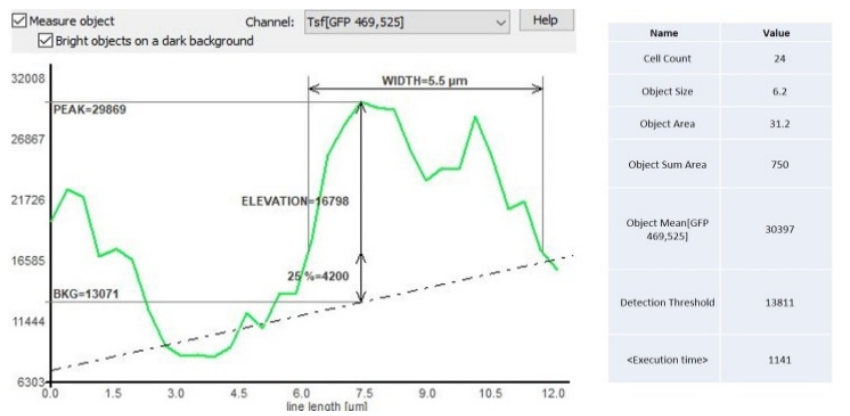

Figure 4: Digital quantification of the demarcated eosinophils: A. Setting the filter and the channel to detect Alexa Fluor 488 wave length, and setting a background cut off value of 13000 pixels to exclude back ground. Intensity of fluorescence was detected at a peak of $\sim 30000$. Digital parameters regarding number of cells, cell size $\sim 6 \mathrm{um}$, average intensity detected are presented in the table.

Table 1: Eosinophils infiltrate in oral squamous cell carcinoma

\begin{tabular}{|l|c|c|c|}
\hline \multirow{2}{*}{$\begin{array}{l}\text { Type of } \\
\text { malignancy }\end{array}$} & \multicolumn{2}{|c|}{ Eosinophils } & \multirow{2}{*}{ Total } \\
\cline { 2 - 3 } & Positive & Negative & \\
\hline $\begin{array}{l}\text { Squamous cell } \\
\text { carcinoma }\end{array}$ & $26(87 \%)$ & $4(13 \%)$ & $30(75 \%)$ \\
\hline Normal & $0(0 \%)$ & $10(100 \%)$ & $10(25 \%)$ \\
\hline Total & $26(65 \%)$ & $14(35)$ & $\begin{array}{c}40 \\
(100 \%)\end{array}$ \\
\hline
\end{tabular}

\section{DISCUSSION}

Tumor inflammation is a field of growing interest in the current era of cancer immunotherapy (17). The stroma of the OSCC can be heavily inflamed and eosinophils have been described as one of the common inflammatory components in OSCC (23). Here we present a method of digital quantification of eosinophils infiltrate in OSCC stromal tissue that can be of significance in measuring the extent of immune suppressive cell infiltrate in OSCC. We show that the Cytation 5 machine is a useful technology in quantifying eosinophils through measuring the eosinophils auto fluorescence, accentuated by Alexa Fluor 488, in FFPE OSCC tumor tissue.

One of the mechanisms for tissue recruitment of eosinophils involves granulocyte monocyte colony stimulating factors (GM-CSF). Interestingly, increase in GM-CSF has been described in peripheral blood of HNSCC (6). Another postulated mechanism is that certain chemotherapy medications has been implicated in blood hypereosinophilia (18).

Eosinophils are an evolutionary preserved cells, that were identified more by staining with acidic stains like eosin (19). Further identification of the cytoplasmic granules crystal core was detected using electron microscopy (9). Eosinophils can be counted in peripheral blood where they represent less than $5 \%$ of the circulating cells, that increases under allergic conditions and parasitic infections (20). Interestingly, eosinophils can be counted in peripheral blood according to their auto fluorescence using flow cytometry. Human eosinophils contain granules rich in cationic proteins characteristic of the effector cells in host defense, in particular, in mediating inflammatory responses in human disease (9). Surface expression of IL-5 receptor subunit $\alpha$ (IL-5R $\alpha$ ), CC-chemokine receptor 3 (CCR3) or sialic acidbinding immunoglobulin like lectin 8 (Siglec-8) (in humans) or Siglec-F (also known as Siglec-5) (in mice) in the absence of other lineage-specific markers can be used to identify eosinophils in flow cytometry. Importantly, certain markers that are often used to identify non-eosinophils (such as CD11C, GR1, F4/80 and MHC class II molecules) are expressed by eosinophils recruited into some tissue sites (9). The detection of the eosinophils cellular biomarkers can be difficult on FFPE tissue due to the auto fluorescence as well as background chromogen on immunohistochemistry. Hence, using the proposed technology of Cyation 5 on FFPE tissue to read eosinophils autofluorescnce in alignment with eosinophils detection using the flowcytometry provides an advantage on FFPE tissue.

Recent attention to Eosinophils as predictive biomarkers in several malignancies including intestinal malignancies, melanoma, and lung adenocarcinoma has been observed (14, $21,22)$. Several studies described eosinophils in squamous cell carcinoma (23-26). A most recent study has described eosinophils in OSCC in correlation to several patient clinicopathological manifestations, in which tumor tissue eosinophilia showed significant statistical correlation with smoking, alcohol, betel nut chewing, and advanced tumor pathological staging (10). A limitation of this study was that it used subjective counting of eosinophils. Another drawback was that the authors described the eosinophils as mononuclear cells with eosinophilic granular cytoplasm (10). In addition to other studies describing quantification of eosinophils in OSCC that was also limited by the subjective counting of the cells (27-30). The current work provides a digital non subjective method of quantification and confirms that the auto-fluorescent cells are bi-nucleated with granular cytoplasm, that was confirmed on the H\&E stained serial section as bi-nucleated cells with granular eosinophilic cytoplasm.

Although histological examination is limited by the fact of examining 5um section of the whole tumor tissue, detecting eosinophils in the tumor tissue carries the advantage of preserving the spatial configuration and relation of the eosinophils to the tumor islands. The application of this methodology to quantify eosinophils can be applied on SCC as well as other malignancies. This study also opens avenues for further studies for correlation of eosinophils infiltrate with underlying allergic or patient infectious conditions (24).Our data contributes to our understanding of the inflammatory profile of OSCC. It highlights eosinophils as an inflammatory cell and as a therapeutic target for immune modulation in OSCC.

\section{CONCLUSION}

The digital quantification of eosinophils in OSCC stroma would provide accurate means of evaluating the extent of immune suppression in a tumor and subsequently better understanding of differential patient response to standard immunotherapy. 


\section{CONFLICT OF INTERSET}

The authors declare that they have no conflicts of interest.

\section{REFERENCES}

1. Seiwert TY, Burtness B, Mehra R, Weiss J, Berger R, Eder JP, et al. Safety and clinical activity of pembrolizumab for treatment of recurrent or metastatic squamous cell carcinoma of the head and neck (KEYNOTE-012): an open-label, multicentre, phase 1b trial. Lancet Oncol2016 Jul;17(7):956-65.

2. Mirza AH, Thomas G, Ottensmeier $\mathrm{CH}$, King EV. Importance of the immune system in head and neck cancer. Head Neck2019 Feb 28.

3. Kagedal A, Rydberg Millrud C, Hayry V, Kumlien Georen S, Lidegran M, Munck-Wikland E, et al. Oropharyngeal squamous cell carcinoma induces an innate systemic inflammation, affected by the size of the tumour and the lymph node spread. Clin Otolaryngol2018 Apr 21.

4. Piva MR, LB DES, Martins-Filho PR, Nonaka CF, T DESS, ES DESA, Piva D. Role of inflammation in oral carcinogenesis (Part II): CD8, FOXP3, TNF-alpha, TGFbeta and NF-kappaB expression. Oncol Lett2013 Jun;5(6):1909-14.

5. Chow LQ, Haddad R, Gupta S, Mahipal A, Mehra R, Tahara M, et al. Antitumor Activity of Pembrolizumab in Biomarker-Unselected Patients With Recurrent and/or Metastatic Head and Neck Squamous Cell Carcinoma: Results From the Phase Ib KEYNOTE-012 Expansion Cohort. J Clin Oncol2016 Sep 19.

6. Pak AS, Wright MA, Matthews JP, Collins SL, Petruzzelli GJ, Young MR. Mechanisms of immune suppression in patients with head and neck cancer: presence of CD34(+) cells which suppress immune functions within cancers that secrete granulocytemacrophage colony-stimulating factor. Clin Cancer Res1995 Jan;1(1):95-103.

7. Ravin KA, Loy M. The Eosinophil in Infection. Clin Rev Allergy Immunol2016 Apr;50(2):214-27.

8. Wen T, Rothenberg ME. The Regulatory Function of Eosinophils. Microbiol Spectr2016 Oct;4(5).

9. Weller PF, Spencer LA. Functions of tissue-resident eosinophils. Nat Rev Immunol2017 Dec;17(12):746-60.

10. De Paz D, Chang KP, Kao HK, Lao WW, Huang YC, Chang YL, et al. Clinical Implications of TumorAssociated Tissue Eosinophilia in Tongue Squamous Cell Carcinoma. Laryngoscope2018 Aug 10.

11. Sassler AM, McClatchey KD, Wolf GT, Fisher SG. Eosinophilic infiltration in advanced laryngeal squamous cell carcinoma. Veterans Administration Laryngeal Cooperative Study Group. Laryngoscope1995 Apr;105(4 Pt 1):413-6.

12. Ono Y, Fujii M, Kameyama K, Otani Y, Sakurai Y, Kanzaki J. Expression of matrix metalloproteinase-1 mRNA related to eosinophilia and interleukin-5 gene expression in head and neck tumour tissue. Virchows Arch1997 Nov;431(5):305-10.

13. Joshi PS, Kaijkar MS. A histochemical study of tissue eosinophilia in oral squamous cell carcinoma using
Congo red staining. Dent Res J (Isfahan)2013 Nov;10(6):784-9.

14. Ye L, Wang H, Li H, Liu H, Lv T, Song $\mathrm{Y}$, et al. Eosinophil peroxidase over-expression predicts the clinical outcome of patients with primary lung adenocarcinoma. J Cancer2019;10(4):1032-8.

15. Gao J, He Y, Wu L. [The clinical analysis of eosinophil infiltration in human squamous cell carcinoma of buccal mucosa]. Hua Xi Kou Qiang Yi Xue Za Zhi1997 Aug;15(3):228-9, 32.

16. Ethier C, Lacy P, Davoine F. Identification of human eosinophils in whole blood by flow cytometry. Methods Mol Biol2014;1178:81-92.

17. Msaouel P, Massarelli E. Immune Checkpoint Therapy in Head and Neck Cancers. Cancer J2016 MarApr;22(2):108-16.

18. Hamdan D, Leboeuf C, Le Foll C, Bousquet G, Janin A. Re-exploring immune-related side effects of docetaxel in an observational study: Blood hypereosinophilia. Cancer Med2019 Mar 10.

19. Kay AB. The eosinophil in infection diseases. J Infect Dis1974 May;129(5):606-13.

20. Simon D, Simon HU. Therapeutic strategies for eosinophilic dermatoses. Curr Opin Pharmacol2019 Feb 8;46:29-33.

21. Kruckel A, Moreira A, Frohlich W, Schuler G, Heinzerling L. Eosinophil-cationic protein - a novel liquid prognostic biomarker in melanoma. BMC Cancer2019 Mar 7;19(1):207.

22. Caruso AA, Costigliola F, Salzano J, Del Prete S, Marasco D, Imperatore C, et al. Nasal and systemic eosinophilia associated with solid intestinal tumors, a case report and review of the literature. Ann Ital Chir2019 Mar 4;8.

23. Tadbir AA, Ashraf MJ, Sardari Y. Prognostic significance of stromal eosinophilic infiltration in oral squamous cell carcinoma. J Craniofac Surg2009 Mar;20(2):287-9.

24. Suzuki K, Inokuchi A, Miyazaki J, Kuratomi Y, Izuhara K. Relationship between squamous cell carcinoma antigen and the clinical severity of allergic rhinitis caused by Dermatophagoides farinae and Japanese cedar pollen. Ann Otol Rhinol Laryngol2010 Jan;119(1):22-6.

25. Saxena S, Singh A, Singh P, Sundaragiri KS, Sankhla B, Bhargava A. Evaluating the Role of Immunological Cells (Tissue Eosinophils and Mast Cells) in Progression of Oral Squamous Cell Carcinoma. Mymensingh Med J2018 Apr;27(2):382-8.

26. Debta P, Debta FM, Chaudhary M, Bussari S. Evaluation of myeloid cells (tumor-associated tissue eosinophils and mast cells) infiltration in different grades of oral squamous cell carcinoma. Indian $\mathrm{J}$ Med Paediatr Oncol2016 Jul-Sep;37(3):158-67.

27. Jain S, Phulari RG, Rathore R, Shah AK, Sancheti S. Quantitative assessment of tumor-associated tissue eosinophilia and mast cells in tumor proper and lymph nodes of oral squamous cell carcinoma. J Oral Maxillofac Pathol2018 May-Aug;22(2):227-33.

28. Vaibhav SL, Priya PL, Sonam CK, Supriya K, Garima Y, Sabeer S, et al. Evaluation of Tumor-associated Tissue Eosinophilia in Different Stages of Oral Squamous Cell 
Carcinoma using Special Stains: An in vitro Histopathological Study. J Contemp Dent Pract2018 May 1;19(5):579-86.

29. Peurala E, Tuominen M, Loyttyniemi E, Syrjanen S, Rautava J. Eosinophilia is a favorable prognostic marker for oral cavity and lip squamous cell carcinoma. APMIS2018 Mar;126(3):201-7.

30. Martinelli-Klay CP, Lombardi T, Mendis B, Soares EG, Salvado F, Courvoisier DS, et al. Tissue eosinophilia in oral intraepithelial neoplasia as a probable indicator of invasion. Oral Dis2018 Mar;24(1-2):103-8.

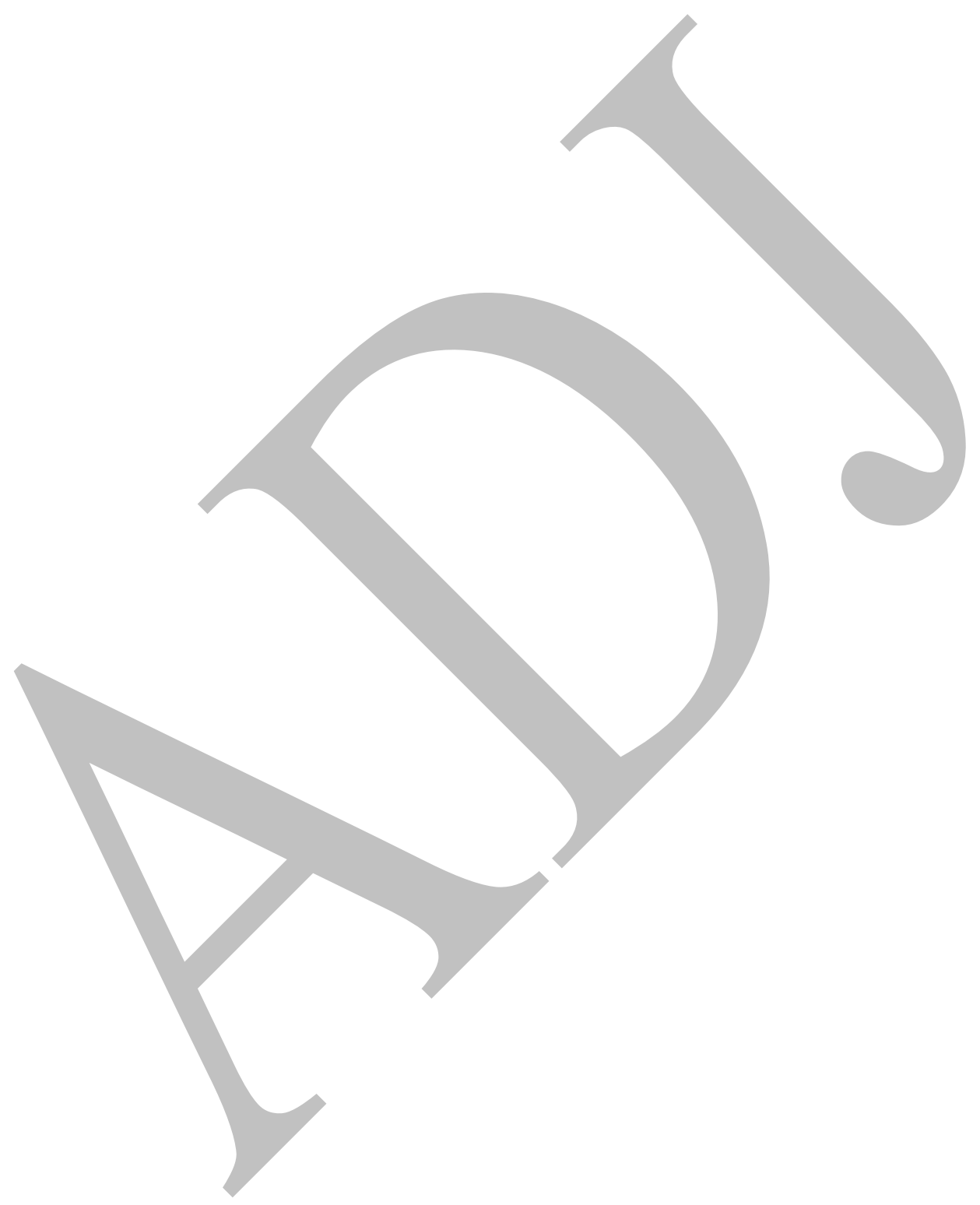

